\title{
The use of educational intervention on cleaning process in a secondary hospital
}

\author{
AF Sola ${ }^{1 *}$, FS Vasques ${ }^{1}$, CCD Oliveira ${ }^{2}$, ACC Pignatari ${ }^{1}$ \\ From 3rd International Conference on Prevention and Infection Control (ICPIC 2015) \\ Geneva, Switzerland. 16-19 June 2015
}

\section{Introduction}

Early in the twentieth century, health professionals became more concerned about the need to provide a clean environment and adherence to aseptic practices when caring for people's health. In that setting, the use of chemical products for cleaning and disinfecting was widespread.For that reason, cleansing is a relevant issue in hospital infection control. By ensuring proper hygiene of hospital items and areas, cross-infection can be reduced.

\section{Objectives}

This study aims to evaluate the technique and effectiveness of the environmental cleansing performed by the hospital hygiene team.

\section{Methods}

This is a qualitative and quantitative prospective study, conducted from January to October 2014 in a 300-bed high complexity hospital, Sao Paulo, Brazil. In this study, we evaluated 2880 items in 360 observations before and after the cleaning team were trained on correct cleaning procedures. Evaluation and measurement were divided in three steps: (a) cleaning technique was monitored by using a visible signaling system with black light and by the measurement of the amount of adenosine trisfofate (ATP) detected on the surfaces. Measurements took place before and after the environmental cleansing;(b) the intervention was applied and the team was trained on correct cleaning procedures.

\section{Results}

During the training period, measurements continued to take place with the same techniques; (c) the cleaning effectiveness was measured again after the training period. A target of $70 \%$ compliance was set by the infection control team. During the study period, the average compliance was $67.97 \%$. Analysis before and during intervention showed $51.69 \%$ and $78.82 \%$ compliance, exceeding the set target.

\section{Conclusion}

In order to have a biologically safe hospital environment it is necessary to work with different environmental peculiarities, establish partnership with different services of the institution and staff, and implement effective actions to control the spread of microorganisms, particularly multi-resistant ones.

\section{Disclosure of interest}

None declared.

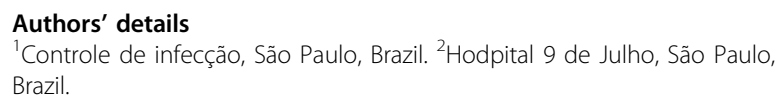

Published: 16 June 2015

\section{doi:10.1186/2047-2994-4-S1-P32}

Cite this article as: Sola et al:: The use of educational intervention on cleaning process in a secondary hospital. Antimicrobial Resistance and Infection Control 2015 4(Suppl 1):P32.

'Controle de infecção, São Paulo, Brazil

Full list of author information is available at the end of the article 